BMJ Open

Diabetes

Research

\& Care

\title{
Effectiveness of plant-based diets in promoting well-being in the management of type 2 diabetes: a systematic review
}

\author{
Anastasios Toumpanakis, ${ }^{1}$ Triece Turnbull, ${ }^{2}$ Isaura Alba-Barba ${ }^{3}$
}

To cite: Toumpanakis A, Turnbull T, Alba-Barba I. Effectiveness of plant-based diets in promoting well-being in the management of type 2 diabetes: a systematic review. BMJ Open Diab Res Care 2018;6:e000534. doi:10.1136/ bmjdrc-2018-000534

Received 8 March 2018 Revised 28 June 2018 Accepted 8 July 2018
Check for updates

\section{(C) Author(s) (or their} employer(s)) 2018. Re-use permitted under CC BY-NC. No commercial re-use. See rights and permissions. Published by BMJ.

${ }^{1}$ School of Health Sciences, University of London, London, UK

${ }^{2}$ Faculty of Health and Society, University of Northampton, Northampton, UK ${ }^{3}$ Department of Cardiology, East Sussex NHS Healthcare Trust, Saint Leonards-on-Sea, UK

Correspondence to Anastasios Toumpanakis; anastasios.toumpanakis@city. ac.uk

\section{ABSTRACT}

Diet interventions have suggested an association between plant-based diets and improvements in psychological well-being, quality of life and glycated hemoglobin (HbA1c) control in populations with diabetes. The aims of this review are to systematically analyze the available literature on plant-based diet interventions targeting diabetes in adults and to clearly define the benefits on well-being of such interventions. This is a systematic review of controlled trials. A computerized systematic literature search was conducted in the following electronic databases: Allied and Complementary Medicine, Cochrane Central Register of Controlled Trials, Cumulative Index to Nursing and Allied Health Literature, E-Journals, Excerpta Medica Database, MEDLINE, Health Management Information Consortium, PsycARTICLES, PsycINF0, PubMed, SocINDEX and Web of Science. The search strategy retrieved 1240 articles, of which 11 met the inclusion criteria $(n=433$; mean sample age 54.8 years). Plant-based diets were associated with significant improvement in emotional well-being, physical well-being, depression, quality of life, general health, HbA1c levels, weight, total cholesterol and low-density lipoprotein cholesterol, compared with several diabetic associations' official guidelines and other comparator diets. Plant-based diets can significantly improve psychological health, quality of life, $\mathrm{HbA1c}$ levels and weight and therefore the management of diabetes.

\section{INTRODUCTION}

\section{Diabetes facts}

WHO estimates that in 2014, 422 million adults were living with diabetes globally, while according to the International Diabetes Federation (IDF) this number will rise to 642 million by $2040 .{ }^{12}$ In the UK the number of people who have diabetes is over 4.5 million, and in the USA it is more than 30 million. ${ }^{2-4}$ In $2010 / 2011$ in the UK, diabetes costed approximately $£ 10$ billion in direct costs and $£ 14$ billion in indirect costs, totaling £24 billion (10\% of the National Health Service budget), with the estimation being $£ 40$ billion in total in $2035 .^{5}$ In the USA the annual economic burden is $\$ 245$ billion, \$176 and \$69 billion in direct and indirect costs, respectively. ${ }^{6}$

Diabetes UK states that $90 \%$ of people with diabetes have type 2 diabetes (T2D), while at the same time the percentage of people with T2D is on the rise and increasing. The increasing levels of obesity in many countries nowadays have underlined a very concerning, newly introduced aspect: the number of T2D in children is growing. ${ }^{4}$ The IDF raises the concern by stating that T2D in children has the potential to become a global public health issue which will lead to serious health outcomes and underlines the need for more research in this aspect of diabetes. ${ }^{2}$

\section{The impact of diabetes}

Diabetes can cause a number of health complications if not well managed and treated and has the potential to have a huge impact on people's physical and psychological wellbeing. The WHO and the IDF suggest that diabetes considerably increases the risk of cardiovascular disease (CVD), nephropathy, loss of vision due to diabetic retinopathy and lower extremity amputation. ${ }^{12}$ Diabetes (and its health complications) is also associated with an increased risk of mortality in most countries. $^{27}$ The IDF estimates the number of people who died from diabetes in 2015 before the age of 60 was approximately 5 million; $14.5 \%$ of all-cause mortality globally is attributed to diabetes. ${ }^{2}$ The countries with the highest number of people with diabetes are the ones with the highest number of deaths associated with diabetes: China, Russian Federation, India and the USA. In 2015 T2D was the seventh leading cause of death in the USA. ${ }^{4}$

T2D is considered to be one of the most psychologically demanding chronic conditions, ${ }^{8}$ and people with diabetes often have 
poor psychological well-being. ${ }^{9}$ Diabetes is often comorbid with depression, which has an impact on its management and control. ${ }^{1}$ A systematic review ${ }^{10}$ shows high rates of comorbidity between diabetes and depression and suggests that depression is three times as common among people with diabetes. The psychological stress associated with the management of diabetes could lead to elevated symptoms of depression. ${ }^{11}$ Depression comorbid with T2D has been associated with poorer glycemic control and poorer management of the condition. ${ }^{12-14}$

\section{Diabetes management}

In January 2018 the American Association of Clinical Endocrinologists and the American College of Endocrinology released their new guidelines in which they suggest that plant-based diet is the optimal nutrition plan for people with diabetes as it promotes the well-being and the better management of diabetes. ${ }^{15}$

The IDF reports that the most influential factor for the development of T2D is lifestyle behavior commonly associated with poor diet (eg, processed and high fat content foods). ${ }^{2}$ WHO underlines the importance of achieving a standardized and consistent management approach by promoting interventions that support healthy diets. ${ }^{1}$

There is a large body of research that suggests the association between high meat consumption and T2D. ${ }^{16-19}$ The European Prospective into Cancer and Nutrition (EPIC) -InterAct study ${ }^{20}$ is a large prospective cohort study which explored the role of lifestyle and genetics on the risk of developing T2D in approximately 340000 adults from eight countries in Europe during a mean period of 11 years. The results of the EPIC-InterAct study ${ }^{20}$ show a high risk of T2D among individuals with high meat consumption, specifically red and processed meat. Moreover, after controlling and managing other risk factors for T2D (eg, smoking, physical activity, alcohol intake), the association between meat consumption and incidence of T2D remained statistically significant. This suggests the importance of healthy diet behavior in the management of T2D.

The term plant-based diet refers to eating habits that avoid the consumption of most or all animal products and support high consumption of fruits, vegetables, legumes, seeds, whole grains and nuts. ${ }^{21}$ Satija $e t$ $a l^{22}$ reviewed three prospective cohort studies of a total sample of 200727 participants and concluded that a plant-based diet is associated with significantly lower risk of T2D. Tonstad $e t a t^{23}$ state that plant-based diets could effectively and substantially decrease diabetes incidents. Furthermore, high consumption of fiber, whole grains, fruits and vegetables is associated with a lower risk of T2D ${ }^{24-27}$ A plant-based diet pattern seems to offer high protection against the development of diabetes as it contains antioxidants, fiber, micronutrients and unsaturated fatty acids, which are considered to act as protective factors against diabetes. ${ }^{22}$ In addition, Mayo ${ }^{28}$ states that the management of diabetes is mostly based on a behavioral approach in which individuals could establish nearly complete control of their condition as long as they have been equipped with the right knowledge and skills. This suggests that effective management of diabetes could result in better glycemic control and psychological well-being.

A meta-analysis ${ }^{29}$ focusing on vegetarian diets (defined as those excluding meat, poultry and fish, and including eggs and dairy) in diabetes revealed a significantly improved glycated hemoglobin (HbA1c) in people who followed a vegetarian diet pattern. No systematic review was found in the literature solely focusing on the psychological and medical outcomes of plant-based diet interventions in adults with T2D.

\section{Objectives}

The aims of this review are to systematically analyze the available literature on plant-based diet interventions targeting and/or including adults with diabetes and to clearly define the benefits on well-being of such interventions.

\section{METHODS}

The incorporation of the Preferred Reporting Items for Systematic Reviews and Meta-Analyses guidelines was used in order to conduct this review. ${ }^{30} 31$ The following criteria were considered for inclusion in the systematic review: (1) plant-based diet interventions targeting or including adults with T2D; (2) age of participants $\geq 18$ years; (3) clearly defined health outcomes; (4) controlled trials with length of $\geq 3$ weeks; and (5) peer-reviewed studies. The exclusion criteria were (1) diet interventions for diabetes other than type 2; (2) age of participants $<18$ years; (3) uncontrolled studies; (4) diet interventions that included more than $10 \%$ of daily calories on animal products; and (5) duplicate results.

\section{Search strategy}

A computerized systematic search was performed on 10 November 2017 in the following electronic databases limited to studies published in English language since the inception of each database: Allied and Complementary Medicine, Cochrane Central Register of Controlled Trials, Cumulative Index to Nursing and Allied Health Literature, E-Journals, Excerpta Medica Database, MEDLINE, Health Management Information Consortium, PsycARTICLES, PsycINFO, PubMed, SocINDEX and Web of Science. In order to search for further potentially eligible research material, the reference lists of studies with relevant topic were searched and reviewed. The following were the search terms used to locate/find studies: (1) plant based OR plant-based OR "plant based" OR plant food OR "plant food" OR vegan* OR vegetarian* OR "Daniel fast"; (2) diabet* OR T2D OR DT2; and (3) intervention* OR program* OR "controlled trial*" OR "randomised controlled trial*" OR "randomized controlled trial*" OR RCT. 


\section{Data extraction}

The following data was collected from each study as available: quality of life, self-esteem, depression,perceived pain and neuropathy symptoms, foot conductance, nutritional efficacy,general efficacy, dietary adherence, dietary restraint, dietary disinhibition, diet acceptability, quality of life HbA1c, weight, fasting blood glucose, total cholesterol, low-density lipoprotein cholesterol, triglycerides anddiabetes-related medication. In addition, baseline characteristics of each study's sample size, mean age, gender (proportion of men), design and duration were collected. Mean values for baseline age, proportion of male population and $\mathrm{HbA1c}$ were also calculated.

\section{Data screening and quality measures}

The search results from the databases were combined and then the identified duplicates were removed. The described inclusion criteria were applied in a standardized manner, and relevant studies were screened using the title, abstract and full text of the article.

The Quality Assessment Tool for Quantitative Studies is a standardized quality appraisal instrument that was developed by the Effective Public Health Practice Project (EPHPP) of McMaster University for assessing the quality of public health. ${ }^{32}$ The instrument has been evaluated for inter-rater reliability, content validity and construct validity and has received high scores. ${ }^{33} 34$ This quality assessment tool and its dictionary are provided at www. ephpp.ca. Two researchers carried out the quality assessment independently. The identified relevant studies were critically assessed according to the EPHPP's tool and rated as 'strong', 'moderate' or 'weak' in six sections: (1) selection bias; (2) study design; (3) confounders; (4) blinding; (5) data collection methods; and (6) withdrawals and dropouts. The aim of the quality assessment was to extract results from studies with an overall 'strong' or 'moderate' methodological rating. Studies that were rated as 'poor' have been excluded from the systematic review. Please see table 1 for the results of quality assessment of included studies.

\section{RESULTS}

\section{Study selection process}

The search strategy retrieved 1240 articles. After removing the duplicates, the title/abstract screening process identified 41 studies. After a further full-text assessment, 30 articles were excluded from the systematic review and 11 met the inclusion criteria (please see figure 1). No additional publications were found through reference lists and hand searching.

The included studies relied on different tools in order to extract psychological data outcomes, and therefore a meta-analysis was not possible in this regard. For the other outcomes of our study, we considered that because the overall pooled $\mathrm{N}$ in the study is small, the CIs would be quite wide due to imprecision, which might cause our pooled estimate to cross the null hypothesis, which would make it difficult to draw a conclusion in either direction. Dietary interventions are difficult to engage a large number of individuals as it is hard for participants to alter their dietary patterns for a medium/long period of time. Our aim was to present as clear as possible the physical and psychological outcomes of plant-based interventions.

\section{Study characteristics}

The 11 included studies were published between 1999 and 2017 (please see table 2). Two of the studies produced two articles from the same sample highlighting different aspects of research; these articles are included:

1. Kahleova et al (2013) used different data from Kahleova et al (2011). ${ }^{35} 36$

2. Barnard et al (2009b) used different data from Barnard et al (2009a). ${ }^{37} 38$

The majority of the studies $(n=6)^{37-42}$ were conducted in the USA, while the rest took place in Czech Republic

Table 1 Quality assessment results for the included studies

\begin{tabular}{|c|c|c|c|c|c|c|c|}
\hline Authors (year) & $\begin{array}{l}\text { Selection } \\
\text { bias }\end{array}$ & $\begin{array}{l}\text { Study } \\
\text { design }\end{array}$ & Confounders & Blinding & $\begin{array}{l}\text { Data } \\
\text { collection } \\
\text { methods }\end{array}$ & $\begin{array}{l}\text { Withdrawal } \\
\text { and } \\
\text { dropouts }\end{array}$ & $\begin{array}{l}\text { Global } \\
\text { rating }\end{array}$ \\
\hline Wright et al ${ }^{44}(2017)$ & Moderate & Strong & Weak & Moderate & Strong & Moderate & Moderate \\
\hline Lee et a $/^{45}$ (2016) & Strong & Strong & Strong & Moderate & Strong & Strong & Strong \\
\hline Bunner et al/1 (2015) & Strong & Strong & Strong & Moderate & Strong & Strong & Strong \\
\hline Soare et $a l^{43}$ (2014) & Strong & Strong & Strong & Moderate & Strong & Strong & Strong \\
\hline Mishra et al ${ }^{39}(2013)$ & Moderate & Strong & Strong & Moderate & Strong & Moderate & Strong \\
\hline Kahleova et a/ ${ }^{35}$ (2013) & Strong & Strong & Strong & Moderate & Strong & Strong & Strong \\
\hline Kahleova et al ${ }^{36}(2011)$ & Strong & Strong & Strong & Moderate & Strong & Strong & Strong \\
\hline Ferdowsian et al ${ }^{40}(2010)$ & Moderate & Strong & Strong & Moderate & Strong & Strong & Strong \\
\hline Barnard et $\left.a\right|^{37}$ (2009b) & Strong & Strong & Strong & Moderate & Strong & Strong & Strong \\
\hline Barnard et a/ ${ }^{38}$ (2009a) & Strong & Strong & Strong & Moderate & Strong & Strong & Strong \\
\hline Nicholson et al/2 (1999) & Strong & Strong & Strong & Moderate & Strong & Moderate & Strong \\
\hline
\end{tabular}



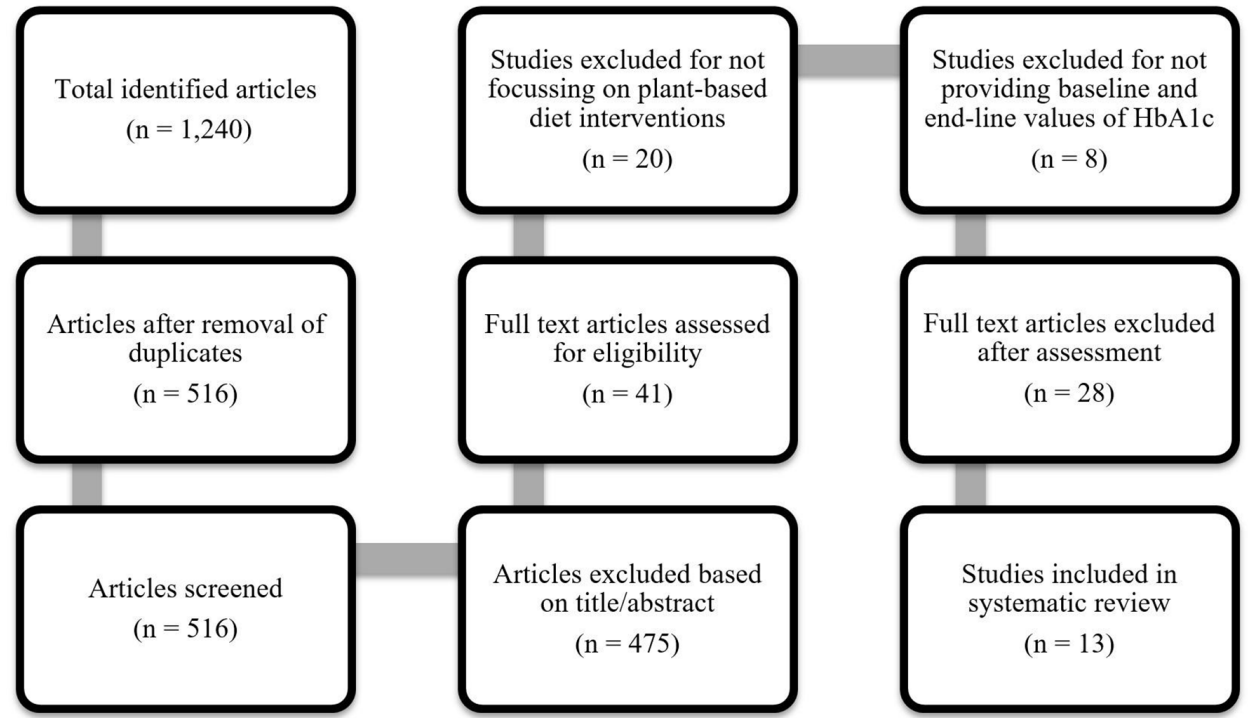

Figure 1 Study selection process.

$(\mathrm{n}=2),{ }^{35}{ }^{36}$ Italy $(\mathrm{n}=1),{ }^{43}$ New Zealand $(\mathrm{n}=1)^{44}$ and South Korea $(n=1){ }^{45}$ One study had been conducted in a supervised residential environment, ${ }^{43}$ while the rest had been conducted in community-based settings. The total sample size was 433 participants (219 in the intervention groups/214 in the control groups; mean sample size 48), aged between 27 and 80 years (mean age 54.8 years). The number of withdrawals was reported in all studies, with the rates being between $3.5 \%$ and $29.1 \%$. All studies targeted populations with T2D except for three. ${ }^{39} 4044$ Of these three studies, two ${ }^{3940}$ included individuals with body mass index (BMI) $\geq 25 \mathrm{~kg} / \mathrm{m}^{2}$ and/or T2D, and one $^{44}$ also included individuals with BMI $\geq 25 \mathrm{~kg} / \mathrm{m}^{2}$ and at least one diagnosis of T2D or ischemic heart disease or hypertension or hypercholesterolemia. Therefore not all of the participants of these three studies had T2D, but in the results specific outcomes related to the individuals with T2D were described.

All included studies were controlled trials with a duration range of between 3 and 74 weeks (mean duration 23.2 weeks). Of the nine controlled trials, seven were randomized controlled trials (RCTs), of which four reported the randomization process ${ }^{38} 414445$ and three failed to describe it. ${ }^{364243}$ Moreover, two studies used a parallel $\operatorname{design}^{3644}$ and one used a cluster study design. ${ }^{39}$ Eight of the studies examined vegan diets in the intervention groups, while one study examined plant-based diet with an option of one portion of low-fat yogurt a day, which accounted for approximately $8 \%$ of the total daily intake of calories. ${ }^{36}$ A full description of the diet intervention was described in all of the articles. All studies reported HbAlc as their primary or secondary outcome, which was measured at baseline and at the end of each study in both groups. From the 11 articles included, 3 reported psychological well-being outcomes in their results. The studies were dietary interventions and therefore none included blinding of participants. Five controlled trials provided nutritional education in both groups ${ }^{38424345}$ and four provided education only to the intervention group. ${ }^{39-41} 44$ All studies reported both baseline and end-line results and used validated measures. Highly specialized professionals (medical doctors, registered dietitians) delivered the dietary interventions in all of the studies.

\section{FINDINGS}

\section{Quality of life, psychological and social well-being}

Quality of life significantly improved only in the intervention $\operatorname{group}^{35} 41 \quad(\mathrm{p}=0.01 ; \mathrm{p}<0.05)$, and significant improvements in the intervention group in both psychological and physical components summary ${ }^{44}(\mathrm{p}<0.001$; $\mathrm{p}<0.0001)$ were also reported. The control group showed a significant improvement only in the physical component summary ${ }^{44}(p=0.03)$. Significant differences between the two groups in favor of the intervention group were reported for both the psychological and physical component summary ${ }^{44}(p<0.01 ; p=0.03)$. Depression levels dropped and reached statistical significance only in the intervention group ${ }^{35}(\mathrm{p}=0.03)$. Changes in perceived pain and neuropathy symptoms were reported as pain significantly decreased between groups favoring the intervention group ${ }^{41}(p=0.04)$. Also, a significant decline in foot conductance was reported in the control group ${ }^{41}$ $(\mathrm{p}=0.03)$, suggesting that the intervention might have stopped or slowed down the progress of nerve impairment. Furthermore, statistically significant differences were reported in favor of the intervention group for self-esteem ${ }^{44}(p<0.01)$, nutritional efficacy ${ }^{44}(p<0.0001)$ and general efficacy ${ }^{44}(\mathrm{p}=0.01)$.

Overall, the results suggest that a plant-based diet could improve the overall quality of life, psychological wellbeing and chronic diabetic neuropathy in people with T2D without changes in food enjoyment and diet costs. In addition, the slight increase in electrochemical skin conductance in the intervention group and the significant decline of the control group ${ }^{41}$ make plant-based diet 


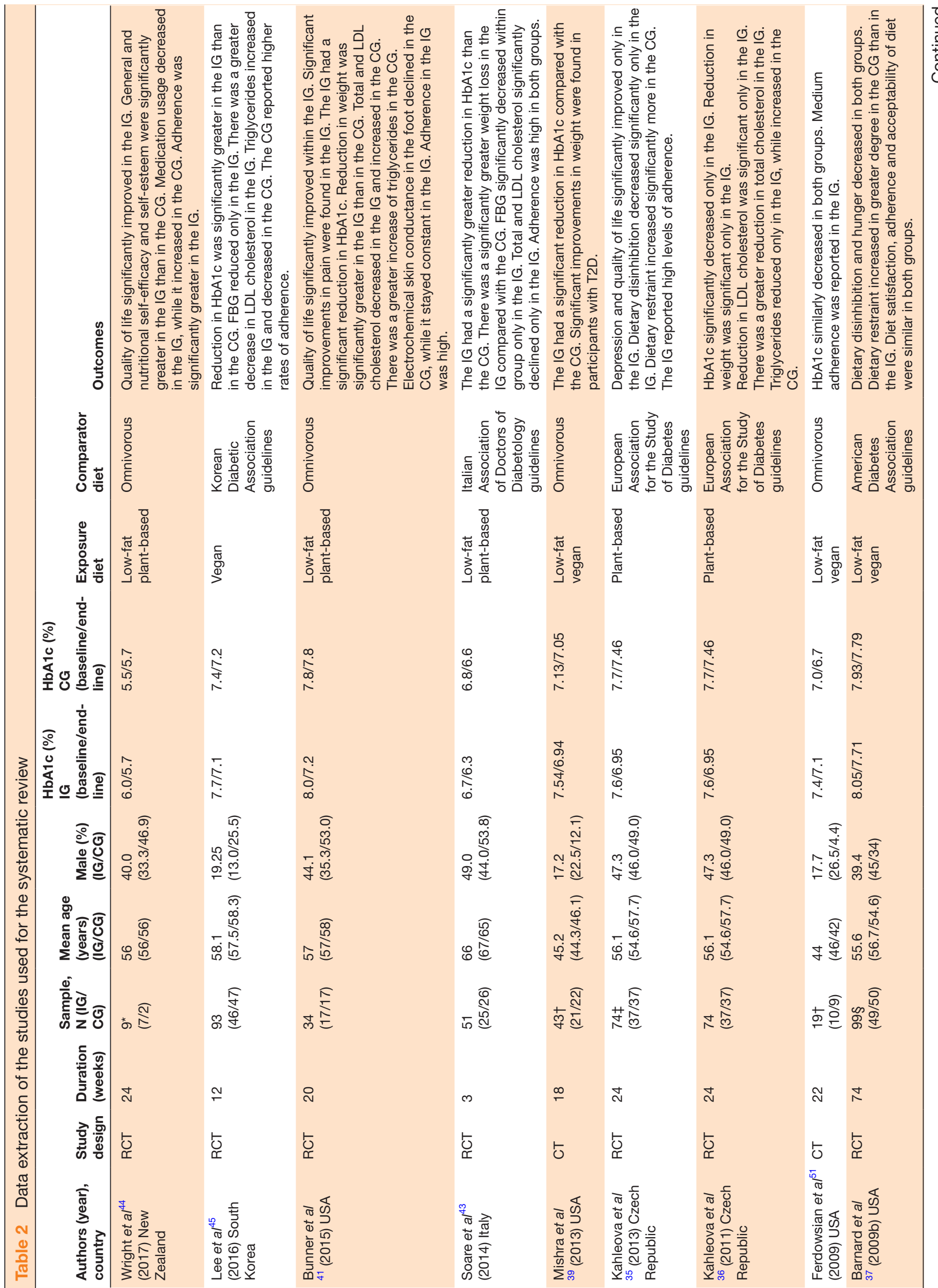




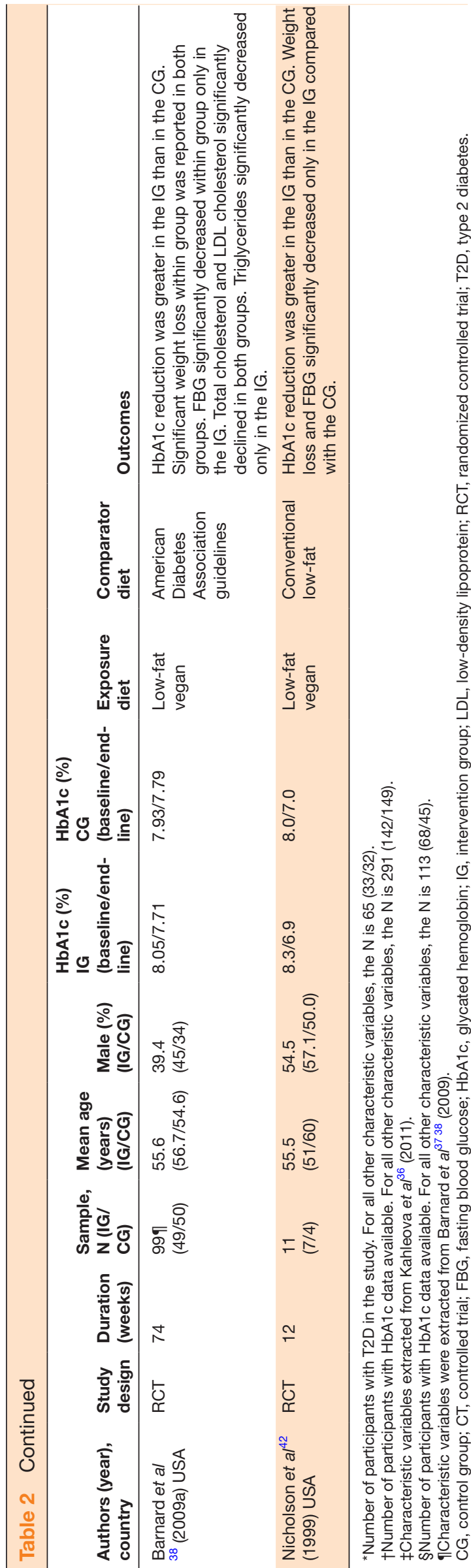

intervention promising for further testing in people with painful T2D neuropathy.

\section{Adherence, acceptability and diet behavior}

Five studies ${ }^{36} 37394144$ reported greater adherence among the participants of the intervention group compared with the control group, while one study ${ }^{43}$ showed high adherence among both groups. Also, one study ${ }^{40}$ reported complete adherence to the plant-based intervention diet in approximately half of participants $(44 \%)$. In contrast, Lee $e t a l^{45}$ reported that the control group reported higher adherence $(\mathrm{p}=0.002)$ compared with the intervention group, while the percentage of participants who reported high adherence was significantly higher in the control group compared with the intervention group $(\mathrm{p}<0.001)$.

Dietary restraint increased to a greater extent only in the control group, ${ }^{35} 37$ suggesting that the control group felt more constrained by their assigned diet than the participants of the plant-based diet group. Dietary disinhibition decreased significantly only in the intervention group ( $\mathrm{p}=0.01)$ in one study, ${ }^{35}$ while Barnard $e t$ $a \hat{l}^{37}$ reported that it declined in both groups, suggesting that individuals in both groups were feeling less hungry compared with baseline and they were less likely to overeat as a response to stressful stimuli. Also, no significant differences between the two groups were reported with regard to food acceptability. ${ }^{37}$ Within-group analysis demonstrated significant improvement in levels of energy only in the intervention group $(\mathrm{p}<0.001),{ }^{37}$ while the control group reported significant improvement in digestion $(\mathrm{p}<0.05){ }^{37}$

Overall, the results showed that even though the plantbased diets were slightly more demanding in initial effort, adherence was greater among those in the intervention groups compared with the control groups, suggesting high rates of acceptability of the plant-based diet among the participants of the intervention groups. In addition, control group diets were more likely to be described as constraining, and as a result dietary restraint was higher in the control groups.

\section{HbA1c and diabetes control}

All nine studies provided baseline and end-line measures for HbAlc. In eight studies the decrease of HbAlc was greater among participants in the intervention groups compared with the control groups, while in one study ${ }^{40}$ the decrease was similar in both groups. After excluding Wright et $a l^{44}$ for reasons of not reporting HbAlc levels specifically for participants with T2D and after performing weighting statistical adjustment, the mean difference among the eight studies that provided specific HbA1c levels for participants with T2D between baseline and end-line measurements shows a decrease of $0.55 \%$ in the intervention groups and $0.19 \%$ in the control groups. The results suggest that participants in the intervention groups managed to better control their diabetes 
via the plant-based diet compared with participants in the control groups.

Further analyses of HbA1c levels in two studies ${ }^{3640}$ among participants whose medication remained unchanged showed a greater decrease of $1 \%$ in the intervention group compared with a decrease of $0.2 \%$ in the control group in one study ${ }^{40}$ and significantly decreased by $0.9 \%$ only in the intervention group $(p=0.002)$ in the other. ${ }^{36}$

In addition, an analysis among participants who reported high adherence showed a greater difference of HbAlc levels between the two groups ${ }^{45}$ : a significant within-group decrease of $0.9 \%$ in the intervention group $(\mathrm{p}<0.01)$ and a significant within-group decrease of $0.3 \%$ in the control group $(p<0.05)$. The reported decrease between the two groups was significantly greater in the intervention group compared with the control group among participants of high adherence $(\mathrm{p}=0.01){ }^{45}$

\section{Further medical outcomes}

Six studies reported a reduction or discontinuation of diabetes-related medications among participants at end-line. ${ }^{36}{ }^{38}{ }^{41-44}$ Interestingly, Nicholson et $a t^{42}$ mentioned that two intervention group participants on insulin decreased their insulin doses from baseline to end-line. Moreover, three intervention group participants decreased and one discontinued the oral hypoglycemic treatment. ${ }^{42}$ Additionally, two participants in the intervention group discontinued the antihypertensive medication at end-line. ${ }^{42}$ In contrast, participants in the control group did not have changes on their medication. ${ }^{42}$ Barnard et $a l^{38}$ reported that $35 \%(\mathrm{n}=17)$ of the participants in the intervention group reduced their T2D medication in contrast to $20 \%(n=10)$ of the participants in the control group. Kahleova $e t a p^{36}$ showed that the diabetic medication reduced in $43 \%$ of participants in the intervention group and in 5\% of participants in the control group. Soare $e t a t^{43}$ mentioned that from a total of seven participants on oral hypoglycemic medication in the intervention group, five of them discontinued their medication. In the control group one participant discontinued the oral hypoglycemic medication treatment. ${ }^{43}$ Similarly, Bunner et $a l^{11}$ reported that 10 participants in the intervention group reduced the glucose-lowering treatment, while in the control group one participant reduced the glucose-lowering medication. Moreover, four intervention group participants decreased the lipid-lowering medication, while no participant reduced it in the control group. ${ }^{41}$ Wright $e t a l^{44}$ reported that two participants with T2D in the intervention group did no longer meet the criteria for T2D diagnosis at end-line.

Two studies reported an increase in diabetes-related medication at end-line, ${ }^{38} 41$ and one ${ }^{45}$ reported that $14 \%$ $(n=7)$ of the participants in the intervention group and $23 \%(\mathrm{n}=12)$ participants of the control group increased the diabetes medication doses. Bunner et $a l^{41}$ showed that two participants in the intervention group and two in the control group increased the glucose-lowering medication. Also, the lipid-lowering medication increased in one intervention group participant and in three control group participants. ${ }^{41}$

Weighting statistical adjustment has been performed for all the mean values that follow. The review indicated a significantly greater weight loss in participants in the intervention groups (mean weight loss was $5.23 \mathrm{~kg}$ ) compared with the control groups (mean weight loss was $2.83 \mathrm{~kg}) .^{36{ }^{3841-43}}$ Four studies ${ }^{36384243}$ showed a significant decrease in fasting blood glucose (FBG) only in the intervention groups, while one study ${ }^{45}$ reported a reduction in FBG only in the intervention group but without meeting statistical significance. The mean FBG decrease was 22.91 $\mathrm{mg} / \mathrm{dL}$ in the intervention groups and $11.58 \mathrm{mg} / \mathrm{dL}$ in the control groups. Total cholesterol declined, with the reduction being greater in the intervention groups (mean decrease was $21.98 \mathrm{mg} / \mathrm{dL}$ ) compared with the control groups (mean decrease was $11.14 \mathrm{mg} / \mathrm{dL}$ ) in four studies, ${ }^{363841} 43$ of which two ${ }^{3643}$ reported significant results in favor of the intervention groups $(p<0.0001$; $\mathrm{p}<0.001)$. In addition, one study ${ }^{41}$ reported an increase in total cholesterol in the control group. Low-density lipoprotein (LDL) cholesterol greatly decreased among participants in the intervention groups (mean reduction was $12.43 \mathrm{mg} / \mathrm{dL}$ ) compared with the control groups (mean reduction was $6.76 \mathrm{mg} / \mathrm{dL}$ ) in five studies, ${ }^{36} 38414345$ of which three $e^{363843}$ showed significant reduction only in the intervention groups $(\mathrm{p}<0.05 ; \mathrm{p}<0.01 ; \mathrm{p}<0.001)$. One study ${ }^{41}$ reported an increase in LDL among participants in the control group. A greater reduction in triglycerides was reported in the intervention groups (mean decline was $12.86 \mathrm{mg} / \mathrm{dL}$ ) compared with the control groups (mean decline was $5.79 \mathrm{mg} / \mathrm{dL}$ ). ${ }^{36} 38414245$ One study $^{38}$ reported a significant reduction $(\mathrm{p}<0.05)$ in triglycerides, while two studies ${ }^{4145}$ reported an increase of triglycerides, of which one ${ }^{41}$ showed an increase in both groups and the other ${ }^{45}$ showed that triglycerides surprisingly were increased in the intervention group and decreased in the control group. In both studies ${ }^{41} 45$ the results did not reach statistical significance.

\section{DISCUSSION}

This systematic review demonstrates evidence that a plantbased diet can significantly improve psychological wellbeing, quality of life, control of T2D measured by HbAlc and a number of physical characteristics (weight loss, FBG, total cholesterol, LDL cholesterol, triglycerides) in people with T2D.

Three studies reported results with regard to psychosocial and quality of life outcomes. ${ }^{354144}$ More specifically, there were significant improvements in the intervention group compared with the control group in depression, ${ }^{35}$ emotional well-being, ${ }^{44}$ physical well-being ${ }^{44}$ and general quality of life. ${ }^{35444}$ Further outcomes showed a significant decrease of reported pain among participants in the intervention group. ${ }^{41}$ The participants of the intervention groups reported greater psychosocial results and an improved quality of life in all three studies. 
Participants' adherence was reported in eight of the studies. ${ }^{36} 37$ 39-41 43-45 Reported adherence was higher among participants of the intervention groups compared with the control groups in four studies. ${ }^{36373944}$ In contrast Lee $e t a t^{45}$ reported that the control group showed significantly higher adherence compared with the intervention group. A potential explanation for this difference might be the absence of weekly workshops, as Lee $e t a l^{45}$ used only phone consultations, while the four studies with high adherence results in the intervention groups carried out weekly educational sessions. One study reported high adherence in both the intervention and the control groups, ${ }^{43}$ potentially because education was provided on a daily basis and the RCT was conducted in a supervised environment. Moreover, two of the studies monitored the adherence only in the intervention group and reported high $^{41}$ and medium levels of adherence. ${ }^{40}$ These results of the systematic review suggest that providing consistent nutrition education can better support people to adapt more effectively to a plant-based diet, as high dietary adherence and compliance is linked with educational interventions. ${ }^{46-48}$

Three studies monitored the diet behavior and diet acceptability among participants in both groups. ${ }^{353739}$ Dietary disinhibition significantly decreased in the intervention group, while dietary restraint significantly increased only in the control group. ${ }^{35}$ Barnard et $a b^{37}$ reported that both diets were equally well accepted by participants in both groups. Kahleova $e t a l^{35}$ results suggested that the plant-based diet was highly accepted by the participants as the dietary restraint and dietary disinhibition were significantly improved in the intervention group.

With regard to diabetes control, this systematic review demonstrates that a plant-based diet intervention can significantly reduce $\mathrm{HbA1c}$ in adults with T2D and potentially reduce diabetes medication. Eight of the included studies reported baseline and end-line HbAlc levels of participants with T2D. ${ }^{3638-4345}$ All eight studies reported a greater reduction of HbAlc levels among participants in the intervention groups compared with the control groups, with an average decrease of $0.55 \%$ in the intervention groups and $0.19 \%$ in the control groups. This result is consistent with the findings of previous research. ${ }^{29}$ Furthermore, findings from six studies reported a reduction or discontinuation of diabetes-related medication in the intervention groups. ${ }^{36} 3841-44$

Five studies reported significant greater weight loss in the intervention group (mean weight loss $5.23 \mathrm{~kg}$ ) compared with the control group (mean weight loss $2.83 \mathrm{~kg}$ ) from baseline to end-line. ${ }^{36} 3841-43$ Three studies reported significant improvements in FBG levels. ${ }^{36} 3842$ Two studies demonstrated a significant decrease in LDL cholesterol levels in the intervention groups, ${ }^{36} 38$ while one study showed a decrease in total cholesterol and triglycerides levels. ${ }^{38}$ There are studies that have demonstrated that a plant-based diet can significantly lower total cholesterol, LDL cholesterol, triglycerides and the overall risk for CVD. ${ }^{49-52}$ This is noteworthy because CVD is one of the main comorbidities of T2D $\mathrm{D}^{53} 54$ and one of the main causes of premature mortality in the diabetic population. ${ }^{55-59}$

The present systematic review has several strengths. It is the first review, to our knowledge, that attempted to summarize the significant psychological outcomes of a plant-based diet intervention in adults with T2D. Furthermore, the included CTs were conducted in several different countries (Czech Republic, Italy, New Zealand, South Korea and USA), which suggests that generalizability of the findings in the UK setting is likely. Also, reviewing CTs which focus on dietary patterns and not isolated nutrients makes the findings of the review easier to be applied to the general population. Finally, when plant-based diets were compared with official guidelines from a number of countries, the plant-based diet was found to be superior. Although this is a preliminary review of evidence specific to people with T2D, other research which includes but is not specific to people with diabetes supports the psychological findings. ${ }^{6061}$

This systematic review also has limitations. The included studies had rather small sample sizes, although they were adequate to estimate significance levels. Another limitation is that two of the studies were not randomized. ${ }^{39} 40$ In addition, adherence in control trials regarding diet is difficult to be followed and measured by researchers because it is highly dependent on participants' recalls, which might be inaccurate.

\section{CONCLUSIONS}

Based on the evidence of the research analysis by this systematic review, it can be concluded that plant-based diets accompanied by educational interventions can significantly improve psychological health, quality of life, HbAlc levels and weight and therefore the management of diabetes. Furthermore, plant-based diets could potentially improve diabetic neuropathic pain and the levels of total cholesterol, LDL cholesterol and triglycerides in T2D.

There is further need of studies to explore the relationship between psychological health, dietary patterns/ behavior and diabetes control. Future studies could explore ways of delivering proper nutritional education in order to support participants to follow healthier dietary patterns.

Acknowledgements We would like to thank Helen Goodman, who provided guidance and assistance with development of the search terms of the literature. We would also like to thank Dr Angela Manning for her valuable input.

Contributors AT, TT and IA-B designed the study. AT and IA-B collected data and conducted data analysis. AT wrote the manuscript. TT and IA-B made substantial contributions to the identification of relevant literature and interpretation of findings, and were involved in drafting the manuscript and revising it critically. All authors have approved the publication of the manuscript.

Funding Publication fees for this study were covered by the City, University of London.

Competing interests None declared. 
Patient consent Not required.

Provenance and peer review Not commissioned; externally peer reviewed.

Data statement No additional data are available.

Open access This is an open access article distributed in accordance with the Creative Commons Attribution Non Commercial (CC BY-NC 4.0) license, which permits others to distribute, remix, adapt, build upon this work non-commercially, and license their derivative works on different terms, provided the original work is properly cited, appropriate credit is given, any changes made indicated, and the use is non-commercial. See: http://creativecommons.org/licenses/by-nc/4.0/

\section{REFERENCES}

1. World Health Organization, 2016. Global report on diabetes. http:// www.who.int/diabetes/global-report/en (cited 10 Nov 2017).

2. International Diabetes Federation. IDF diabetes atlas seventh edition. Brussels: International Diabetes Federation, 2015.

3. Diabetes UK, 2015. Facts and stats. https://www.diabetes.org.uk/ Professionals/Position-statements-reports/Statistics (cited 10 Nov 2017).

4. Centers for Disease Control and Prevention, 2017. National diabetes statistics report. https://www.cdc.gov/diabetes/pdfs/data/statistics/ national-diabetes-statistics-report.pdf (cited 10 Nov 2017).

5. Hex N, Bartlett C, Wright D, et al. Estimating the current and future costs of Type 1 and Type 2 diabetes in the UK, including direct health costs and indirect societal and productivity costs. Diabetic Medicine 2012;29:855-62.

6. American Diabetes Association. Economic costs of diabetes in the U.S. in 2012. Diabetes Care 2013;36:1033-46.

7. Kirkman MS, Briscoe VJ, Consensus Development Conference on Diabetes and Older Adults. Diabetes in older adults: a consensus report. J Am Geriatr Soc 2012;60:2342-56.

8. Habtewold TD, Islam MA, Radie YT, et al. Comorbidity of depression and diabetes: an application of biopsychosocial model. Int J Ment Health Syst 2016;10:74

9. Mental Health Network. Investing in emotional and psychological wellbeing for patients with long-term conditions. London: NHS Confederation, 2012.

10. Roy T, Lloyd CE. Epidemiology of depression and diabetes: a systematic review. J Affect Disord 2012;142:S8-S21.

11. Golden $\mathrm{SH}$, Lazo M, Carnethon $\mathrm{M}$, et al. Examining a bidirectional association between depressive symptoms and diabetes. JAMA 2008;299:2751-9.

12. Lloyd CE, Pambianco G, Orchard TJ. Does diabetes-related distress explain the presence of depressive symptoms and/or poor self-care in individuals with Type 1 diabetes? Diabet Med 2010;27:234-7.

13. Fisher L, Skaff MM, Mullan JT, et al. A longitudinal study of affective and anxiety disorders, depressive affect and diabetes distress in adults with Type 2 diabetes. Diabet Med 2008;25:1096-101.

14. Lin EH, Katon W, Von Korff M, et al. Relationship of depression and diabetes self-care, medication adherence, and preventive care. Diabetes Care 2004;27:2154-60.

15. Garber AJ, Abrahamson MJ, Barzilay Jl, et al. Consensus statement by the american association of clinical endocrinologists and american college of endocrinology on the comprehensive type 2 diabetes management algorithm - 2018 executive summary. Endocr Pract 2018;24:91-120.

16. Pan $A$, Sun $Q$, Bernstein AM, et al. Changes in red meat consumption and subsequent risk of type 2 diabetes mellitus: three cohorts of US men and women. JAMA Intern Med 2013;173:1328-35.

17. Micha R, Michas G, Mozaffarian D. Unprocessed red and processed meats and risk of coronary artery disease and type 2 diabetes--an updated review of the evidence. Curr Atheroscler Rep 2012;14:515-24.

18. Pan A, Sun Q, Bernstein AM, et al. Red meat consumption and risk of type 2 diabetes: 3 cohorts of US adults and an updated metaanalysis. Am J Clin Nutr 2011;94:1088-96.

19. Aune D, Ursin G, Veierød MB, Ursin VMB. Meat consumption and the risk of type 2 diabetes: a systematic review and meta-analysis of cohort studies. Diabetologia 2009;52:2277-87.

20. Bendinelli B, Palli D, Masala G, et al. Association between diet and type 2 diabetes: EPIC-InterAct study. Diabetologia 2013;56:47-59.

21. McMacken M, Shah S. A plant-based diet for the prevention and treatment of type 2 diabetes. J Geriatr Cardiol 2017;14:342-54.

22. Satija A, Bhupathiraju SN, Rimm EB, et al. Plant-based dietary patterns and incidence of Type 2 diabetes in US men and women: results from three prospective cohort studies. PLoS Med 2016;13:e1002039.
23. Tonstad S, Stewart K, Oda K, et al. Vegetarian diets and incidence of diabetes in the Adventist Health Study-2. Nutr Metab Cardiovasc Dis 2013;23:292-9.

24. Kuijsten A, Aune D, InterAct Consortium. Dietary fibre and incidence of type 2 diabetes in eight European countries: the EPIC-InterAct Study and a meta-analysis of prospective studies. Diabetologia 2015;58:1394-408.

25. Cooper AJ, Forouhi NG, InterAct Consortium. Fruit and vegetable intake and type 2 diabetes: EPIC-InterAct prospective study and meta-analysis. Eur J Clin Nutr 2012;66:1082-92.

26. Ye EQ, Chacko SA, Chou EL, et al. Greater whole-grain intake is associated with lower risk of type 2 diabetes, cardiovascular disease, and weight gain. J Nutr 2012;142:1304-13.

27. Yu R, Woo J, Chan R, et al. Relationship between dietary intake and the development of type 2 diabetes in a Chinese population: the Hong Kong Dietary Survey. Public Health Nutr 2011;14:1133-41.

28. Mayo P. Prevention and management of comorbid diabetes and depression. Nurs Stand 2015;30:46-54

29. Yokoyama Y, Barnard ND, Levin SM, et al. Vegetarian diets and glycemic control in diabetes: a systematic review and meta-analysis. Cardiovasc Diagn Ther 2014;4:373-82.

30. Harms M. The EQUATOR Network and the PRISMA Statement for the reporting of systematic reviews and meta-analyses. Physiotherapy 2009;95:237-40.

31. Liberati A, Altman DG, Tetzlaff $\mathrm{J}$, et al. The PRISMA statement for reporting systematic reviews and meta-analyses of studies that evaluate healthcare interventions: explanation and elaboration. BMJ 2009;339:b2700.

32. National Collaborating Centre for Methods and Tools. Quality assessment tool for quantitative studies. Hamilton: McMaster University, 2008.

33. Armijo-Olivo S, Stiles CR, Hagen NA, et al. Assessment of study quality for systematic reviews: a comparison of the cochrane collaboration risk of bias tool and the effective public health practice project quality assessment tool: methodological research. J Eval Clin Pract 2012;18:12-18.

34. Thomas BH, Ciliska D, Dobbins M, et al. A process for systematically reviewing the literature: providing the research evidence for public health nursing interventions. Worldviews Evid Based Nurs 2004;1:176-84.

35. Kahleova $\mathrm{H}$, Hrachovinova $\mathrm{T}$, Hill $\mathrm{M}$, et al. Vegetarian diet in type 2 diabetes--improvement in quality of life, mood and eating behaviour. Diabet Med 2013;30:127-9.

36. Kahleova $\mathrm{H}$, Matoulek $\mathrm{M}$, Malinska $\mathrm{H}$, et al. Vegetarian diet improves insulin resistance and oxidative stress markers more than conventional diet in subjects with Type 2 diabetes. Diabet Med 2011;28:549-59.

37. Barnard ND, Gloede L, Cohen J, et al. A low-fat vegan diet elicits greater macronutrient changes, but is comparable in adherence and acceptability, compared with a more conventional diabetes diet among individuals with type 2 diabetes. J Am Diet Assoc 2009b;109:263-72.

38. Barnard ND, Cohen J, Jenkins DJ, et al. A low-fat vegan diet and a conventional diabetes diet in the treatment of type 2 diabetes: a randomized, controlled, 74-wk clinical trial. Am J Clin Nutr 2009a;89:1588S-96.

39. Mishra S, Xu J, Agarwal U, et al. A multicenter randomized controlled trial of a plant-based nutrition program to reduce body weight and cardiovascular risk in the corporate setting: the GEICO study. Eur J Clin Nutr 2013;67:718-24.

40. Ferdowsian HR, Barnard ND, Hoover VJ, et al. A multicomponent intervention reduces body weight and cardiovascular risk at a GEICO corporate site. Am J Health Promot 2010;24:384-7.

41. Bunner AE, Wells CL, Gonzales J, et al. A dietary intervention for chronic diabetic neuropathy pain: a randomized controlled pilot study. Nutr Diabetes 2015;5:e158.

42. Nicholson AS, Sklar M, Barnard ND, et al. Toward improved management of NIDDM: a randomized, controlled, pilot intervention using a lowfat, vegetarian diet. Prev Med 1999;29:87-91.

43. Soare A, Khazrai YM, Del Toro R, et al. The effect of the macrobiotic $\mathrm{Ma}-\mathrm{Pi} 2$ diet vs. the recommended diet in the management of type 2 diabetes: the randomized controlled MADIAB trial. Nutr Metab 2014;11:39.

44. Wright N, Wilson L, Smith M, et al. The BROAD study: A randomised controlled trial using a whole food plant-based diet in the community for obesity, ischaemic heart disease or diabetes. Nutr Diabetes 2017;7:e256.

45. Lee YM, Kim SA, Lee IK, et al. Effect of a brown rice based vegan diet and conventional diabetic diet on glycemic control of patients with Type 2 diabetes: a $12-$ week randomized clinical trial. PLoS One 2016;11:e0155918. 
46. Sun Y, You W, Almeida F, et al. The effectiveness and cost of lifestyle interventions including nutrition education for diabetes prevention: a systematic review and meta-analysis. J Acad Nutr Diet 2017;117:404-21.

47. Askari F, Rabiei S, Rastmanesh R. The effects of nutrition education and diet therapy on glycemic and lipidemic control in Iranian patients with Type 2 Diabetes. Journal of Obesity and Weight Loss Therapy 2013;3.

48. Sharifirad G, Entezari MH, Kamran A, et al. The effectiveness of nutritional education on the knowledge of diabetic patients using the health belief model. J Res Med Sci 2009;14:1-6.

49. Dinu M, Abbate R, Gensini GF, et al. Vegetarian, vegan diets and multiple health outcomes: a systematic review with meta-analysis of observational studies. Crit Rev Food Sci Nutr 2017;57:3640-9.

50. Jenkins DJ, Wong JM, Kendall CW, et al. Effect of a 6-month vegan low-carbohydrate ('Eco-Atkins') diet on cardiovascular risk factors and body weight in hyperlipidaemic adults: a randomised controlled trial. BMJ Open 2014;4:e003505.

51. Ferdowsian HR, Barnard ND. Effects of plant-based diets on plasma lipids. Am J Cardiol 2009;104:947-56.

52. Jenkins DJA, Popovich DG, Kendall CWC, et al. Effect of a diet high in vegetables, fruit, and nuts on serum lipids. Metabolism 1997:46:530-7.

53. Health and Social Care Information Centre \& Diabetes UK. National diabetes audit 2011-2012, report 2: complications and mortality. London: Health and Social Care Information Centre, 2013.

54. Long AN, Dagogo-Jack S. Comorbidities of diabetes and hypertension: mechanisms and approach to target organ protection. J Clin Hypertens 2011;13:244-51.
55. Kato M, Noda M, for the JPHC Study Group. Diagnosed diabetes and premature death among middle-aged Japanese: results from a large-scale population-based cohort study in Japan (JPHC study). BMJ Open 2015;5:e007736.

56. Peters SA, Huxley RR, Woodward M. Diabetes as risk factor for incident coronary heart disease in women compared with men: a systematic review and meta-analysis of 64 cohorts including 858,507 individuals and 28,203 coronary events. Diabetologia 2014;57:1542-51.

57. Nwaneri C, Cooper H, Bowen-Jones D. Mortality in type 2 diabetes mellitus: magnitude of the evidence from a systematic review and meta-analysis. Br J Diabetes Vasc Dis 2013;13:192-207.

58. Rao Kondapally Seshasai S, Kaptoge S, Emerging Risk Factors Collaboration. Diabetes mellitus, fasting glucose, and risk of causespecific death. N Engl J Med 2011;364:829-41.

59. Saydah S, Tao M, Imperatore G, et al. GHb level and subsequent mortality among adults in the U.S. Diabetes Care 2009;32:1440-6.

60. Agarwal U, Mishra S, Xu J, et al. A multicenter randomized controlled trial of a nutrition intervention program in a multiethnic adult population in the corporate setting reduces depression and anxiety and improves quality of life: the GEICO study. Am J Health Promot 2015;29:245-54.

61. Katcher HI, Ferdowsian HR, Hoover VJ, et al. A worksite vegan nutrition program is well-accepted and improves healthrelated quality of life and work productivity. Ann Nutr Metab 2010;56:245-52. 